\title{
Analysis of water transport in a five-layer model of PEMFC
}

\author{
Falin Chen ${ }^{\mathrm{a}}$, Min-Hsing Chang ${ }^{\mathrm{b}, *}$, Chi-Fu Fang ${ }^{\mathrm{a}}$ \\ ${ }^{a}$ Institute of Applied Mechanics, National Taiwan University, Taipei 107, Taiwan, ROC \\ ${ }^{\mathrm{b}}$ Department of Mechanical Engineering, Tatung University, Taipei 104, Taiwan, ROC \\ Received 6 August 2006; received in revised form 24 October 2006; accepted 25 October 2006 \\ Available online 4 December 2006
}

\begin{abstract}
The water transport in a proton exchange membrane fuel cell (PEMFC) is investigated in this study. A five-layer theoretical model is proposed that includes anode and cathode gas diffusion layers (GDLs), catalyst layers (CLs), and the layer of proton exchange membrane. Especially, the volume of membrane is assumed to be variable with its water content and this effect on water transport is examined. Both steady and transient transport phenomena are considered by changing several crucial system parameters such as the relative humidity of reactant gas, the porosity of GDL, and the membrane thickness. The results show that if the humidification of the reactant gases is sufficient, the water management would be better for larger porosities of GDLs or a thinner membrane, and the resistance and overvoltage of the membrane can be reduced significantly as well. Furthermore, it is found that the membrane swelling effect will increase the water content of the membrane especially in the region close to the cathode interface, and lengthen the response time for a PEMFC to reach steady state as switching between two different operating conditions in comparison with the case ignoring this effect.
\end{abstract}

(C) 2006 Elsevier B.V. All rights reserved.

Keywords: Water transport; PEMFC; Five-layer model

\section{Introduction}

Proton exchange membrane fuel cell (PEMFC) has received much attention in the past decades due to its widely promising applications especially in vehicles and portable electronic equipments. However, many critical issues still need to be improved in the development of PEMFC for being used as a clean and efficient power system. One of them is the complicated water management problem. It is known that the performance of a PEMFC depends strongly on the water balance within the cell. Therefore, it is important to understand the transport phenomena of water in the PEMFC and its influences on cell performance.

Numerous studies have devoted to developing theoretical models to describe the water transport in PEMFCs [1-15]. Springer et al. [1] presented an one-dimensional steady-state model for a PEMFC based on experimentally determined transport parameters. Bernardi and Verbrugge [2] derived a mathematical model for the cathode side of a PEMFC and found the water transport is a complicated function of the cell operat-

\footnotetext{
* Corresponding author. Tel.: +886 2 25925252x3410; fax: +8862 25997142 E-mail address: mhchang@ so-net.net.tw (M.-H. Chang).
}

ing conditions. They further presented a full cell model [3] and applied it to investigate the mechanisms of water transport. A 2D model of transport was proposed by Fuller and Newman [4] and they utilized this model to examine the water management problems in a PEMFC. Nguyen and White [5] also developed a steady-state 2D model to investigate the effectiveness of various humidification designs. Okada et al. [6] studied the water transport at the anode side and gave a linear transport equation based on the diffusion of water and electroosmotic water drag to analyze the water concentration profiles. Gurau et al. [7] considered the variations of the concentrations and the partial pressures in the gas channels and developed a 2D model for the entire sandwich of a PEMFC. They further derived a halfcell model for the cathode side and obtained rigorous analytical solutions which account for the liquid water content in the gas diffusion layer (GDL) [8]. A 2D model without external humidification of the reactant gases was also derived by Hsing and Futerko [9]. Baschuk and $\mathrm{Li}$ [10] considered the effect of variable degree of water flooding in the cathode side and formulated a model to evaluate its influence on cell performance. Um et al. [11] presented a transient multidimensional model to simulate multicomponent transport in a PEMFC and explore hydrogen dilution effects in the anode feed. Djilali and Lu [12] focused on 
the modeling of non-isothermal and non-isobaric effects and performed an analysis for the cell performance and water transport over a range of operating current densities. A quasi-3D model of water transport in PEMFCs was proposed by Kulikovsky [13], in which the non-linear diffusion of liquid water in the membrane was investigated. Siegel et al. [14] derived a comprehensive 2D model that includes multicomponent and multiphase transport both along the gas channel and through the membrane electrode assembly (MEA). Their results illustrate the importance of water transport within the cell. Recently, a summarized review of fundamental models for PEMFCs was given by Wang [15], in which the current status of water transport research in PEMFCs was extensively introduced.

So far most of the theoretical models concentrated on the analyses under steady-state conditions. However, the transient transport behaviors in a PEMFC are still quite important especially in the application of mobile systems. Wang and Wang [16] developed a 3D transient model to study the transient dynamics of a PEMFC. They further performed numerical simulations for a single channel PEMFC undergoing a step increase in current density [17]. Their results elucidated the profound interactions between the cell voltage response and water transport dynamics in a low-humidity PEMFC. Besides, it is well known that the properties and water content of the membrane play important roles in the factors dominating the cell performance. Several research efforts have been dedicated to the investigation for water management problems in the membrane [18-30]. It has been pointed out by Divisek et al. [25] that a dry membrane will swell in water and the membrane volume (or thickness) depends on its water content. They found experimentally that this property produces a significant effect on the water transport characteristics in the membrane. Nevertheless, only the mathematical model developed by Springer et al. [1] ever took this effect into consideration.
In this study, we develop a one-dimensional mathematical model to describe the water transport phenomena in a PEMFC with a five-layer structure, in which consists of anode and cathode GDLs and catalyst layers (CLs), and the proton exchanger membrane as shown in Fig. 1. Both steady state and transient cell operation conditions are investigated with the consideration for the swelling effect of the membrane caused by the membrane hydration. The results provide more physical insights into the water transport behaviors of a PEMFC and benefit the practical application and design work as well.

\section{Mathematical model}

The entire system domain is shown in Fig. 1. The humidified reactant gases enter the anodic and cathodic gas channels with water concentration $C_{\mathrm{in}, \mathrm{a}}$ and $C_{\mathrm{in}, \mathrm{c}}$, respectively. The water concentration $C$ is defined as the mole number per unit volume in unit ' $\mathrm{mol} \mathrm{cm}{ }^{-3}$ '. To investigate the water transport behavior in the system, some assumptions are made to develop the mathematical model as list below:

1. The system is assumed to be isothermal.

2. Electrochemical reactions occur only in the CLs and the generation of product water in the cathode CL is in gas phase and allowed to be in supersaturated state.

3. The gas mixtures with water vapor in anode and cathode act as ideal gases.

4. The water transport in GDLs and CLs is mainly by diffusion.

5. The membrane can swell with a higher hydration. Hence, the membrane thickness is variable with its water content.

Note that in a PEMFC the water transport is strongly coupled with the thermal management [12] and the non-isothermal condition is particularly important for the flow along the gas channel.

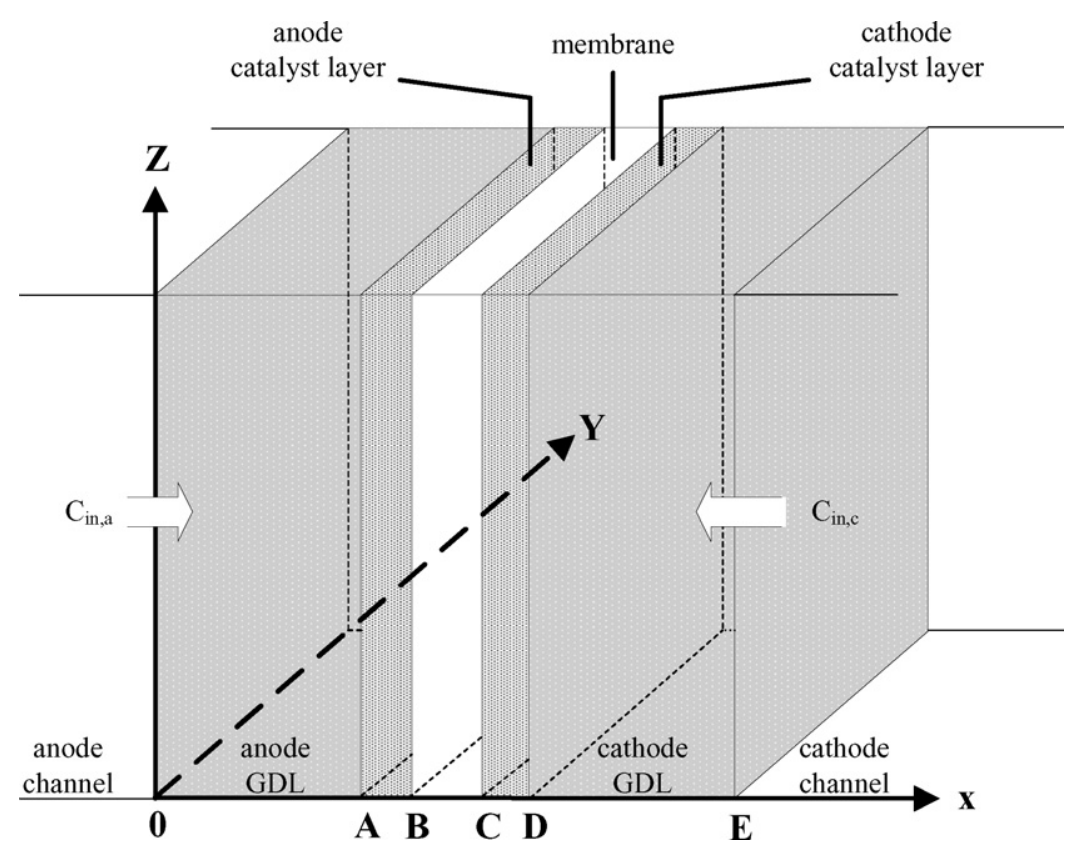

Fig. 1. Schematic diagram of computational domain. 
However, due to the small temperature difference between the fuel cell stack and the operating environment, the isothermal assumption is still reasonable and widely used in many mathematical models [1-5], which have provided many useful insights and predictions of the cell performance. Accordingly, the mathematical model for water transport in each layer can be derived in the following sections.

\subsection{Water transport in the gas diffusion layers}

In general, the mean pore radius of GDL is much larger than the mean free path of gas molecules. In anode side, the main mechanism of gas transport is thus the binary diffusion of hydrogen and water vapor. Although binary diffusion is generally described by Stefan-Maxwell formulas, the equations following these formulas are quite complicated and difficult to handle in computation. It has been found [13] that a single Fick's formula with the effective diffusion coefficient $D_{\mathrm{gl}}^{1}$ can be used instead and give practically the same results. Therefore, the governing equation for water flux $J_{1}\left(\mathrm{~mol} \mathrm{~cm}^{-2} \mathrm{~s}^{-1}\right)$ is given by

$J_{1}=-D_{\mathrm{gl}}^{1} \frac{\partial C_{1}^{\mathrm{g}}}{\partial x}$,

where $C_{1}^{\mathrm{g}}$ is the water concentration in the anode GDL. The parameter $D_{\mathrm{gl}}^{1}$ can be determined by

$\frac{\varepsilon_{\mathrm{d}}^{1.5}}{D_{\mathrm{gl}}^{1}}=\sum_{j} \frac{\xi_{j}}{D_{\mathrm{w} j}}$,

where $\varepsilon_{\mathrm{d}}$ is the porosity of anode GDL, $\xi_{j}$ the mole fraction of the $j$ th component, and $D_{\mathrm{w} j}$ is the binary diffusion coefficient of water vapor in the gas mixture with the $j$ th species. The details for how to determine $D_{\mathrm{wj}}$ can be found in Ref. [31]. The mass conservation equation can be expressed as

$\varepsilon_{\mathrm{d}} \frac{\partial C_{1}^{\mathrm{g}}}{\partial t}=-\frac{\partial J_{1}}{\partial x}, \quad 0<x<A$.

Similarly, the water flux and mass conservation equations in the cathode GDL are respectively

$J_{5}=-D_{\mathrm{gl}}^{5} \frac{\partial C_{5}^{\mathrm{g}}}{\partial x}$

$\varepsilon_{\mathrm{d}} \frac{\partial C_{5}^{\mathrm{g}}}{\partial t}=-\frac{\partial J_{5}}{\partial x}, \quad D<x<E$

where $C_{5}^{\mathrm{g}}$ and $J_{5}$ are the water concentration and flux in the cathode GDL, respectively. Here we assume both anode and cathode GDLs have the same porosity. However, the transport of water vapor in cathode GDL is a multi-component diffusion, in which the effective diffusivity $D_{\mathrm{gl}}^{5}$ can be determined by the equation

$D_{\mathrm{gl}}^{5}=D_{\mathrm{gl}} \varepsilon_{\mathrm{d}}^{1.5}$

where $D_{\mathrm{gl}}$ is the diffusivity in a non-porous condition. Since the order of $D_{\mathrm{gl}}^{1}$ is generally between $10^{-1}$ and $10^{-2} \mathrm{~cm}^{2} \mathrm{~s}^{-1}$ [13]
Table 1

The parameters used in the base case

\begin{tabular}{llc}
\hline Parameter & Symbol & Value \\
\hline Pressure $(\operatorname{atm})$ & $P$ & 3 \\
Temperature $(\mathrm{K})$ & $T$ & 353 \\
Universal gas constant $\left(\mathrm{J} \mathrm{mol}^{-1} \mathrm{~K}^{-1}\right)$ & $R$ & 8.314 \\
Faraday constant $\left(\mathrm{C} \mathrm{mol}^{-1}\right)$ & $F$ & 96,485 \\
Porosity of gas diffusion layer & $\varepsilon_{\mathrm{d}}$ & 0.4 \\
Porosity of catalyst layer & $\varepsilon_{\mathrm{c}}$ & 0.3 \\
Density of dry membrane $\left(\mathrm{g} \mathrm{cm}^{-3}\right)$ & $\rho_{\mathrm{m}}$ & 1.98 \\
Equivalent weight of membrane $\left(\mathrm{g} \mathrm{mol}^{-1}\right)$ & $M_{\mathrm{m}}$ & 1,100 \\
Membrane thickness $(\mu \mathrm{m})$ & $L_{3}$ & 175 \\
Thickness of gas diffusion layer $(\mu \mathrm{m})$ & $L_{1}, L_{5}$ & 300 \\
Thickness of catalyst layer $(\mu \mathrm{m})$ & $L_{2}, L_{4}$ & 20 \\
Effective diffusivity of water vapor $\left(\mathrm{cm}^{2} \mathrm{~s}^{-1}\right)$ & $D_{\mathrm{gl}}^{1}, D_{\mathrm{gl}}^{5}$ & $1.8 \times 10^{-2}$ \\
Effective diffusivity of water vapor $\left(\mathrm{cm}^{2} \mathrm{~s}^{-1}\right)$ & $D_{\mathrm{cl}}^{2}, D_{\mathrm{cl}}^{4}$ & $1.2 \times 10^{-2}$ \\
\hline
\end{tabular}

and the value of $D_{\mathrm{gl}}^{5}$ estimated from the work [3] is also approximately within the same order, we may simply assume $D_{\mathrm{gl}}^{1}=D_{\mathrm{gl}}^{5}$ in the numerical computations. The values of parameters $\varepsilon_{\mathrm{d}}$ and $D_{\mathrm{gl}}^{1}$ at the base case are list in Table 1.

\subsection{Water transport in the catalyst layers}

The catalyst layer contains voids and electrolyte phase. Hence, three mechanisms may contribute to water transport in CLs. In voids the water vapor is transported by Knudsen diffusion, while in the electrolyte phase the water transport is mainly due to diffusion and drag. The flux in the electrolyte phase is related to a correction factor that accounts for the amount of electrolyte in the CL and can be approximated by the volume fraction of the electrolyte phase. Since the correction factor is generally quite small [13] which makes the water transport due to the electrolyte phase be negligible, we can assume the water transport is primarily in vapor form through the voids due to Knudsen diffusion. Accordingly, at the anode CL we have

$J_{2}=-D_{\mathrm{cl}}^{2} \frac{\partial C_{2}^{\mathrm{g}}}{\partial x}$

where $J_{2}$ and $C_{2}^{\mathrm{g}}$ are, respectively, the water flux and water concentration in this layer, and $D_{\mathrm{cl}}^{2}$ is the Knudsen diffusion coefficient of water vapor in the voids that is defined by

$D_{\mathrm{cl}}^{2}=\psi \bar{r}\left(\frac{8 R T}{\pi M_{\mathrm{w}}}\right)^{1 / 2}$.

In above equation, $\psi$ is the correction factor, $\bar{r}$ the mean pore radius, $R$ the universal constant, $T$ the absolute temperature, $M_{\mathrm{W}}$ the molecular weight of water, and the square root the mean thermal velocity of water molecules. Substitute Eq. (7) into the mass conservation equation, we have

$\varepsilon_{\mathrm{c}} \frac{\partial C_{2}^{\mathrm{g}}}{\partial t}=-\frac{\partial J_{2}}{\partial x}, \quad A<x<B$, 
where $\varepsilon_{\mathrm{c}}$ is the porosity of CL. Similarly, for the CL in cathode side, the corresponding water flux $J_{4}$ satisfies

$J_{4}=-D_{\mathrm{cl}}^{4} \frac{\partial C_{4}^{\mathrm{g}}}{\partial x}$.

However, since we assume the generation of water vapor in this layer only, the water transport equation of this layer takes the form

$\varepsilon_{\mathrm{c}} \frac{\partial C_{4}^{\mathrm{g}}}{\partial t}=-\frac{\partial J_{4}}{\partial x}+\frac{S_{\mathrm{w}} Q}{n F}, \quad C<x<D$,

where $S_{\mathrm{w}}$ is the stoichiometry factor of water, $Q$ the electrochemical reaction rate, $n$ the number of electrons, and $F$ is the Faraday constant. Here we also assume both CLs have the same porosity and diffusion coefficient.

\subsection{Water transport in the membrane}

Assume liquid water exists in the bulk membrane, the water transport in the membrane is primarily contributed by three mechanisms: diffusion, electroosmotic drag, and convective motion due to pressure gradient. Recently, it has been found experimentally [13] that the influence of convective motion is quite limited and plays a minor role only on water transport in the membrane. Therefore, we consider the effects of diffusion and electroosmotic drag only and the governing equation of water flux $J_{3}$ can be written as

$J_{3}=-D_{\mathrm{m}} \frac{\partial C_{3}^{1}}{\partial x}+\frac{n_{\mathrm{d}} i}{F}$,

where $D_{\mathrm{m}}$ and $C_{3}^{\mathrm{l}}$ are, respectively, the diffusion coefficient and concentration of liquid water, $n_{\mathrm{d}}$ the drag coefficient, and $i$ the current density. The value of $n_{\mathrm{d}}$ is assumed to be linearly proportional to the water content $\lambda$ in the relationship [1]

$n_{\mathrm{d}}=0.1136 \lambda$,

where $\lambda$ is defined as the ratio of the number of water molecules to the number of charge $\left(\mathrm{SO}_{3}{ }^{-} \mathrm{H}^{+}\right)$sites. The value of $\lambda$ depends on the water vapor activity $a[1]$, and the water uptake curve of the membrane that describes the variation of $\lambda$ with $a$ from a detailed fit of experimental data used in Refs. [1,32] is adopted here for simulating purposes and given by

$\lambda=0.043+17.81 a-39.85 a^{2}+36.0 a^{3}$.

Note that the diffusion coefficient $D_{\mathrm{m}}$ is assumed to relate a water flux by the gradient of the logarithm of activity [1] with respect to the water content $\lambda$ in the form

$D_{\mathrm{m}}=D^{\prime} \frac{\mathrm{d}(\ln a)}{\mathrm{d} \lambda}$

and in order to account for the effect of membrane swelling in the model, the liquid water concentration $C_{3}^{1}$ is expressed in terms of the equivalent water concentration of a dry membrane, $\lambda \rho_{\mathrm{m}}^{\mathrm{d}} / M_{\mathrm{m}}$, by expanding the dry membrane thickness with the factor $(1+s \lambda)$ in the form [1]

$C_{3}^{1}(1+s \lambda)=\frac{\lambda \rho_{\mathrm{m}}^{\mathrm{d}}}{M_{\mathrm{m}}}$.

Thus, the diffusion part of the water flux in Eq. (12) can be written by

$J_{3, \text { diff }}=-D^{\prime}\left(\frac{\lambda \rho_{\mathrm{m}}^{\mathrm{d}} / M_{\mathrm{m}}}{a(1+s \lambda)^{2}} \frac{\mathrm{d} a}{\mathrm{~d} \lambda}\right) \frac{\partial \lambda}{\partial x}$,

where $\rho_{\mathrm{m}}^{\mathrm{d}}$ is the density of dry membrane, $M_{\mathrm{m}}$ the equivalent weight of the membrane, and $s$ is the membrane expansion coefficient. The diffusion coefficient $D^{\prime}$ is relative to $\lambda$ and the functional relationship between $D^{\prime}$ and $\lambda$ shown in Fig. 3 of Ref. [1] is used in the present study. Some detailed discussions for the determination of $D_{\mathrm{m}}$ can be found in the studies [1,32]. Substitute Eq. (12) into the mass conservation equation, we obtain

$\frac{\partial C_{3}^{1}}{\partial t}=-\frac{\partial J_{3}}{\partial x}, \quad B<x<C$,

which is the governing equation of water transport in the membrane.

\subsection{Boundary and initial conditions}

The initial and boundary conditions at each interface in the system are introduced in sequence below. For the initial conditions, we assume the water concentration in each layer at time $t=0$ is denoted by

$C_{j}^{i}(x, 0)=C_{j, 0}^{i}(x)$

where the subscript $j$ stands for the number of each layer and the superscript $i$ denotes the phase of water. For the boundary conditions, we assume the water concentrations at the interfaces $x=0$ and $x=E$ are the same as those of the humidified reactant gases, respectively. Thus, we have

$C_{1}^{\mathrm{g}}=C_{\mathrm{in}, \mathrm{a}}$,

$C_{5}^{\mathrm{g}}=C_{\text {in }, \mathrm{c}}$

At the interface between the GDL and the CL, $x=A$ and $x=D$, the continuities of water concentration and flux result in the following conditions

$C_{1}^{\mathrm{g}}=C_{2}^{\mathrm{g}}$,

$D_{\mathrm{gl}} \frac{\partial C_{1}^{\mathrm{g}}}{\partial x}=D_{\mathrm{cl}} \frac{\partial C_{2}^{\mathrm{g}}}{\partial x}$,

and

$C_{4}^{\mathrm{g}}=C_{5}^{\mathrm{g}}$,

$D_{\mathrm{cl}} \frac{\partial C_{4}^{\mathrm{g}}}{\partial x}=D_{\mathrm{gl}} \frac{\partial C_{5}^{\mathrm{g}}}{\partial x}$, 
As for the conditions at the catalyst-membrane interfaces, we consider the water penetration into the membrane from the gas phase [27] and then at the anode side we have

$k\left(C_{\mathrm{a}}-C_{3}^{1}\right)=-D_{\mathrm{m}} \frac{\partial C_{3}^{1}}{\partial x}+\frac{n_{\mathrm{d}} i}{F}$,

where $k$ is the mass transfer coefficient for the liquid water entry in accordance with the Henry's law penetration, and $C_{\mathrm{a}}$ can be determined by the local vapor concentration [13] via the relationship

$C_{\mathrm{a}}=C_{\mathrm{H}^{+}} \Lambda\left(\frac{\left.C_{2}^{\mathrm{g}}\right|_{x=B}}{C_{\mathrm{sat}}}\right)$,

where $C_{\mathrm{H}^{+}}$is the mole concentration of protons in the membrane, $C_{\mathrm{sat}}$ the mole concentration of saturated water vapor, and the definition of function $\Lambda$ can be found in Ref. [13]. Similarly, the boundary conditions at the cathode side can be written as

$k\left(C_{\mathrm{c}}-C_{3}^{\mathrm{l}}\right)=D_{\mathrm{m}} \frac{\partial C_{3}^{1}}{\partial x}-\frac{n_{\mathrm{d}} i}{F}$,

$C_{\mathrm{c}}=C_{\mathrm{H}^{+}} \Lambda\left(\frac{\left.C_{4}^{\mathrm{g}}\right|_{x=C}}{C_{\mathrm{sat}}}\right)$.

The governing equations together with the initial and boundary conditions are solved numerically with appropriate system parameters. Some typical values of these parameters used in computations are list in Table 1 as the base case. Once the water content of the membrane $\lambda$ is obtained, the membrane conductivity $\sigma$ also can be determined by the fitting result [1] as function of $\lambda$

$\sigma(\lambda)=(0.005139 \lambda-0.00326) \exp \left[1268\left(\frac{1}{303}-\frac{1}{T}\right)\right]$, and then the membrane resistance $R_{\mathrm{m}}$ can be calculated by integration over the membrane thickness $L_{3}$ as

$R_{\mathrm{m}}=\int_{0}^{\mathrm{L}_{3}} \frac{\mathrm{d} x}{\sigma(\lambda)}$.

Accordingly, the overvoltage caused by the membrane resistance can be simply determined by

$\phi=i R_{\mathrm{m}}$

where $\phi$ is the membrane overvoltage.

\section{Results and discussion}

We first discuss the results for the cell operating under steady state conditions, and then elucidate the transient characteristics when the cell operates between two different states. Fig. 2 shows the profiles of water concentration ratio $C / C_{\mathrm{sat}}$ in both anodic and cathode sides and water content $\lambda$ in the membrane. Both anode and cathode reactant gases are humidified with saturated water vapor that $C_{\mathrm{in}, \mathrm{a}} / C_{\mathrm{sat}}$ and $C_{\mathrm{in,c}} / C_{\mathrm{sat}}$ are equal to $100 \%$. As shown in Fig. 2(a), the water vapor is transported from the anode gas channel to the membrane through the GDL and CL for the four assigned values of current density $I=0.25,0.5,0.75$, and $1.0 \mathrm{~A} \mathrm{~cm}^{-2}$. It is found that all these curves decrease gradually and the experience an abrupt jump to lower values across the CL. The higher transport resistance of CL is induced by its lower vapor diffusivity. But the extra vapor contained in the humidified intake gas still reaches the membrane to prevent the anode side of the membrane becoming dried out. In the membrane as shown in Fig. 2(b), the maximum of water content occurs at the cathode boundary because of the generation of water there, and then $\lambda$ decreases gradually toward the anode side. At low current density conditions, for example, in the case $I=0.25 \mathrm{~A} \mathrm{~cm}^{-2}$, the water content is relatively lower at the cathode boundary while decreases slowly to the anode side resulting
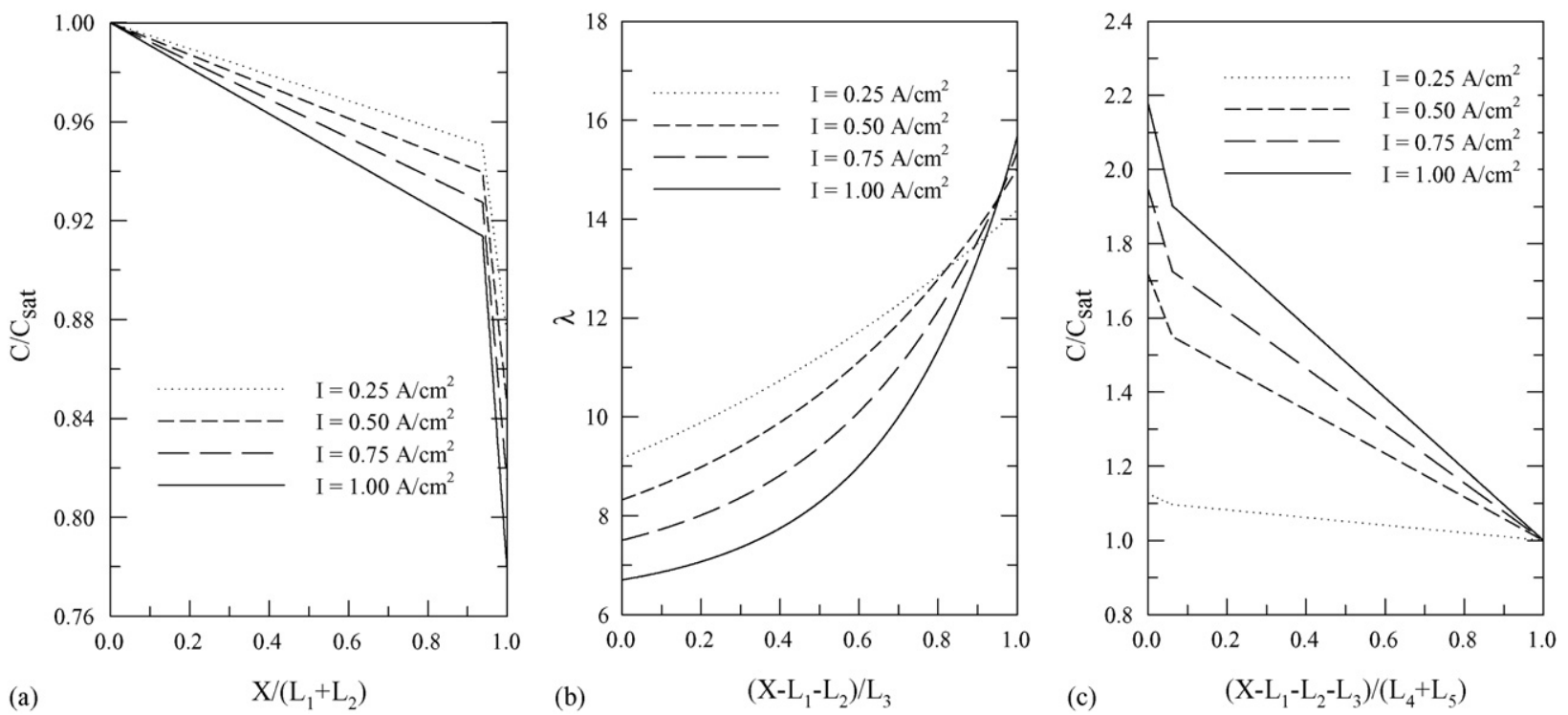

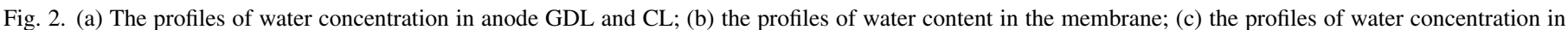
cathode GDL and CL; with four assigned current density $I$, where $C_{\mathrm{in}, \mathrm{a}} / C_{\mathrm{sat}}=C_{\mathrm{in}, \mathrm{c}} / C_{\mathrm{sat}}=100 \%, \varepsilon_{\mathrm{d}}=0.4, L_{3}=175 \mu \mathrm{m}$, and $s=0.0126$ 
in a higher average water content in the membrane. In contrast to this case, at high current density condition as shown in the typical case $I=1.0 \mathrm{~A} \mathrm{~cm}^{-2}$, the water content is higher at the right interface but descends quickly to the left side causing a lower average water content in the membrane. Note that the water transport in the membrane is dominated mainly by two mechanisms, the electroosmotic drag and the back diffusion from cathode to anode. Obviously, when the cell operates at lower current densities, the effect of back diffusion prevails and thus the anode boundary possesses higher water content. But if the cell operates at higher current densities, the effect of electroosmotic drag becomes significant and reduces the water content at the anode boundary distinctly. These results are consistent with the experimental observations [30] that the membrane suffers from the drying out problem especially at the anode side. As indicated by Fig. 2(a), the saturated humidified intake gas indeed helps the membrane prevent from drying out but the water transport resistance increase obviously with the current density. Therefore, it is quite important to reduce the transport resistance across the anode GDL and CL especially at high current density conditions. In the CL and GDL of the cathode side as shown in Fig. 2(c), because of the generation of water vapor inside the $\mathrm{CL}$, the water concentration always appears to have a maximum at the membrane/CL interface and then decreases gradually toward the cathode gas channel for all cases considered. In the case $I=0.25 \mathrm{~A} \mathrm{~cm}^{-2}$, the water concentration in the $\mathrm{CL}$ is only slightly higher than that of the intake saturated air, which indicates that the flooding phenomena should be limited. However, as the current density increases to $I=1.0 \mathrm{~A} \mathrm{~cm}^{-2}$, the water concentrations in the CL and GDL rise rapidly and the high supersaturated concentration implies that the condensation of water vapor may become a severe problem to block the pores of the CL and GDL, and reduce the amount of oxygen transport.

When the relative humidity in both anode and cathode reactant gases reduce to $80 \%$, the profiles of water concentration in each layer are demonstrated in Fig. 3(a)-(c) for four typical cases with current densities $I=0.25,0.5,0.75$, and $1.0 \mathrm{~A} \mathrm{~cm}^{-2}$. As seen in Fig. 3(a), it is found that at lower current densities $I=0.25$ and $0.5 \mathrm{~A} \mathrm{~cm}^{-2}$, the water concentrations of the anode intake gas are less than those at the anode side of membrane. Thus, the corresponding curves increase gradually in the GDL and than jump to higher values across the CL. The transport of water vapor is toward to the anode gas channel and produces an effect of drying out of the membrane. But in the cases $I=0.75$ and $1.0 \mathrm{~A} \mathrm{~cm}^{-2}$, because the effect of electroosmotic drag becomes dominant and diminishes the water content at the anode side of the membrane, both curves still decrease in the GDL and CL, and humidify the anode side of the membrane.

The profiles of water content in the membrane as shown in Fig. 3(b) are similar to Fig. 2(b), except that the water content is lower than that in Fig. 2(b) at the same current density. In Fig. 3(c), we observe that the water concentration in cathode CL and GDL still rises gradually with operating current density, but the water concentration at the cathode side of membrane now is slightly less than $100 \%$ which indicates the flooding phenomena due to condensation of water vapor is insignificant in this situation. These results also suggest that a lower humidified gas around $80 \%$ in cathode gas channel is helpful to maintain appropriate water concentration in the cathode side of membrane. We also find that if the humidity in both anode and cathode gas channels further reduces to $60 \%$, all the four cases exhibit the same behaviors to dry out the membrane at the anode side and the mean water content of membrane reduces significantly as well as the water concentration at the interface between the membrane and the cathode CL.

The porosity of GDL is also an important factor to affect the water transport in the system. Fig. 4 shows the results as the porosities of both anode and cathode GDLs reduce to 0.2 while the other parameters are the same as those considered in Fig. 2. Obviously, a smaller porosity raises the transport resistance
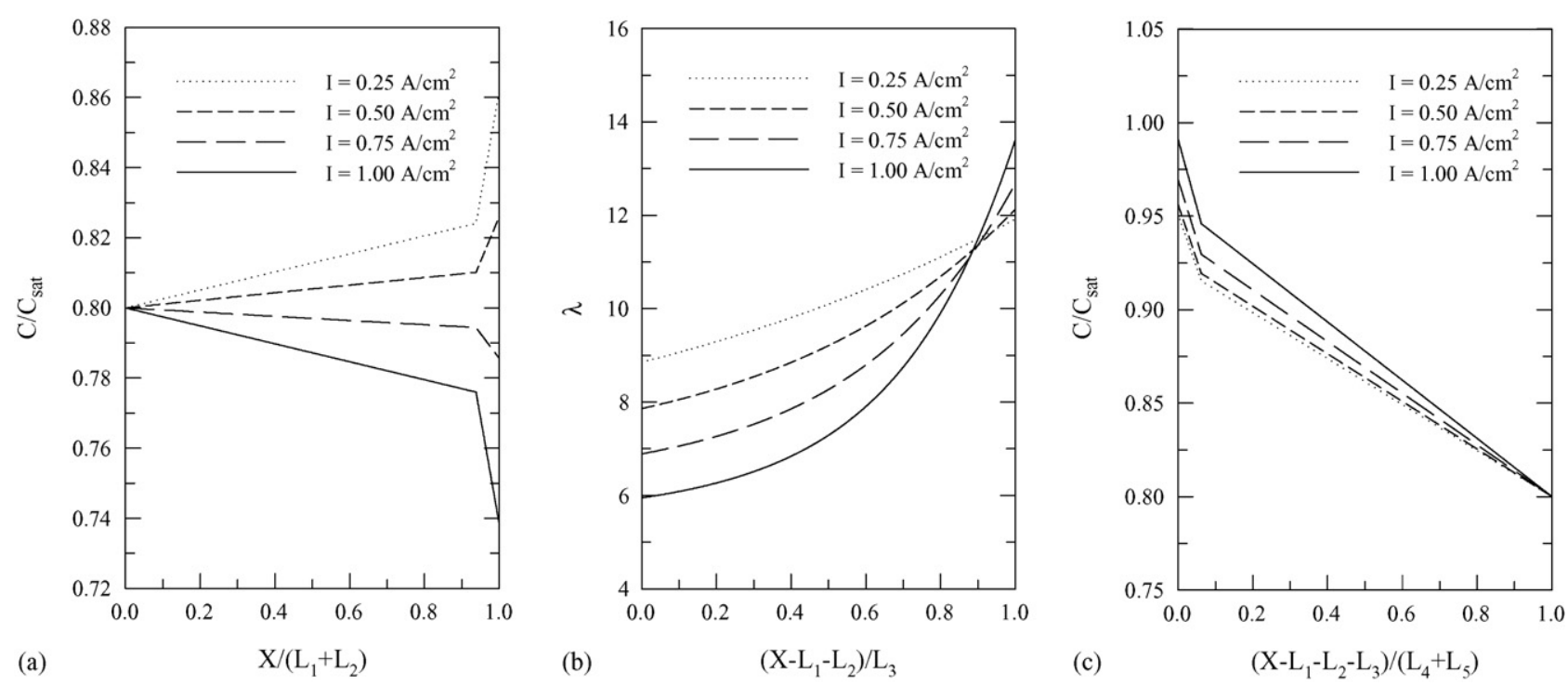

Fig. 3. (a) The profiles of water concentration in anode GDL and CL; (b) the profiles of water content in the membrane; (c) the profiles of water concentration in cathode GDL and CL; with four assigned current density $I$, where $C_{\mathrm{in}, \mathrm{a}} / C_{\mathrm{sat}}=C_{\mathrm{in}, \mathrm{c}} / C_{\mathrm{sat}}=80 \%, \varepsilon_{\mathrm{d}}=0.4, L_{3}=175 \mu \mathrm{m}$, and $s=0.0126$. 

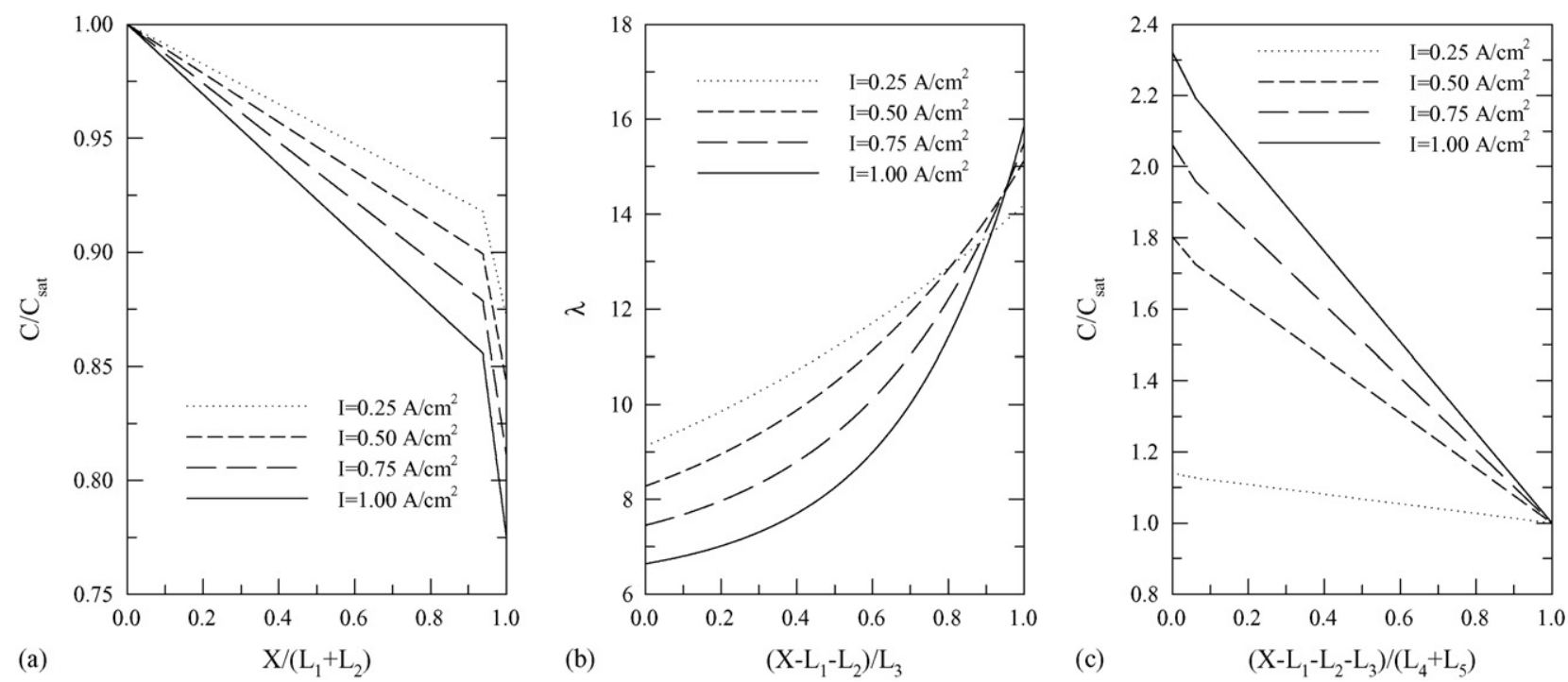

Fig. 4. (a) The profiles of water concentration in anode GDL and CL; (b) the profiles of water content in the membrane; (c) the profiles of water concentration in cathode GDL and CL; with four assigned current density $I$, where $C_{\mathrm{in}, \mathrm{a}} / C_{\mathrm{sat}}=C_{\mathrm{in}, \mathrm{c}} / C_{\mathrm{sat}}=100 \%, \varepsilon_{\mathrm{d}}=0.2, L_{3}=175 \mu \mathrm{m}$, and $s=0.0126$.

through GDL resulting in a larger water concentration difference across it. As seen in Fig. 4(a), all the curves descend more rapidly and reach lower water concentrations at the GDL/CL interface. The profiles of water content of the membrane also rise especially at the right interface between with the cathode CL. Since the water vapor generates in cathode CL, an increase of transport resistance apparently causes a higher water concentration at the membrane/CL interface as shown in Fig. 4(c). As a result, the flooding problem due to vapor condensation may become more serious in the case with a smaller porosity of GDL.

To explore the influence of membrane thickness on water transport, we reduce the membrane thickness $L_{3}$ to $150 \mu \mathrm{m}$ and keep the other parameters the same as those used in Fig. 2. The results are demonstrated in Fig. 5(a)-(c). Apparently, the back diffusion effect becomes more pronounced in a thinner membrane. As shown in Fig. 5(b), the water content at the left interface of membrane increases significantly and that at the right interface reduces slightly in comparison with the results of Fig. 2(b). The water concentration of cathode CL also decreases simultaneously as shown in Fig. 5(c).

The influence of membrane swelling on its water content is examined in Fig. 6 for several assigned values of membrane expansion coefficient $s$ at $I=1.0 \mathrm{~A} \mathrm{~cm}^{-2}$. It is known that a hydrated membrane will swell in comparison with a dry membrane and the ion channel for the transport of ions will be enlarged simultaneously. Therefore, the number of water molecules per charged $\left(\mathrm{SO}_{3}{ }^{-} \mathrm{H}^{+}\right)$site will increase for a more hydrated membrane especially when the swelling effect becomes more significant and $\lambda$ can go as high as 28 at the cathode side when $s=0.0426$ as shown in Fig. 6 . One can see that
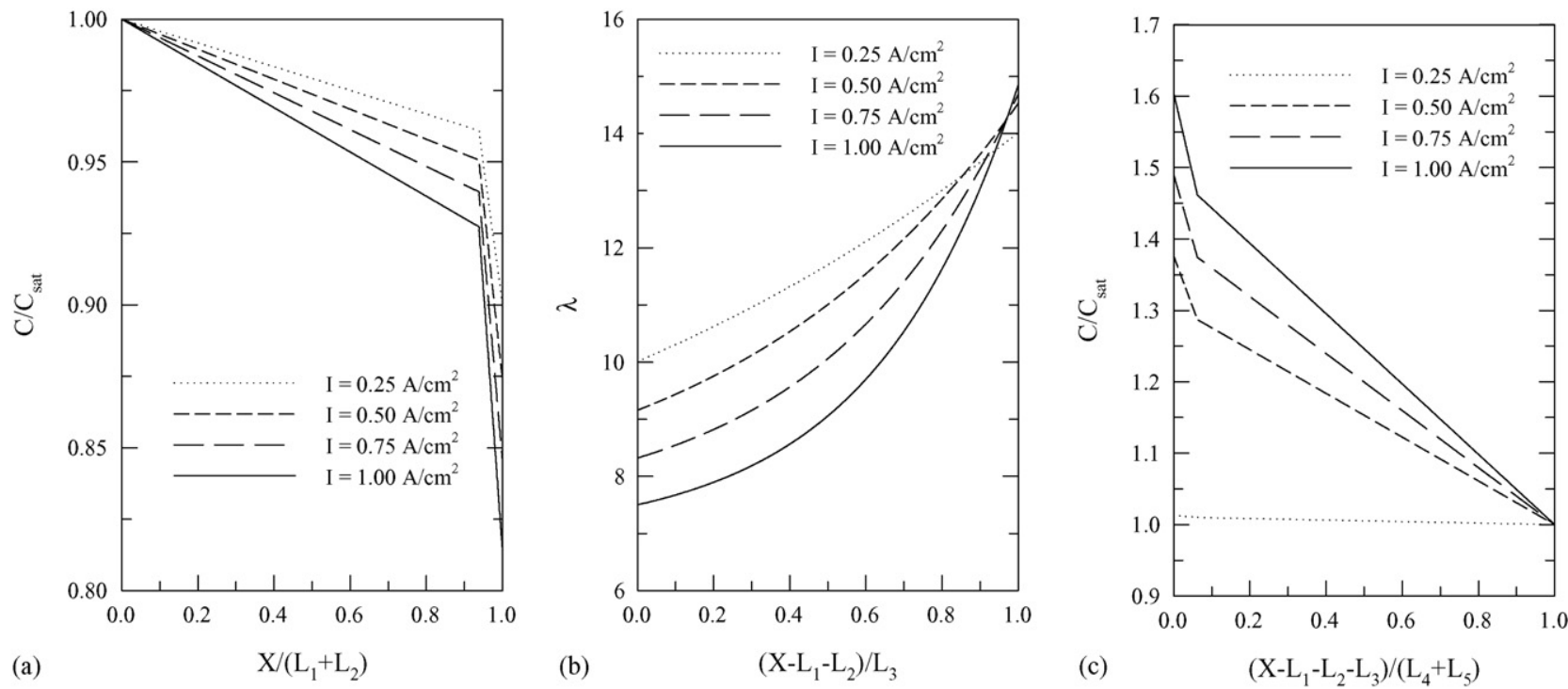

Fig. 5. (a) The profiles of water concentration in anode GDL and CL; (b) the profiles of water content in the membrane; (c) the profiles of water concentration in cathode GDL and CL; with four assigned current density $I$, where $C_{\mathrm{in}, \mathrm{a}} / C_{\mathrm{sat}}=C_{\mathrm{in}, \mathrm{c}} / C_{\mathrm{sat}}=100 \%, \varepsilon_{\mathrm{d}}=0.4, L_{3}=150 \mu \mathrm{m}$, and $s=0.0126$. 


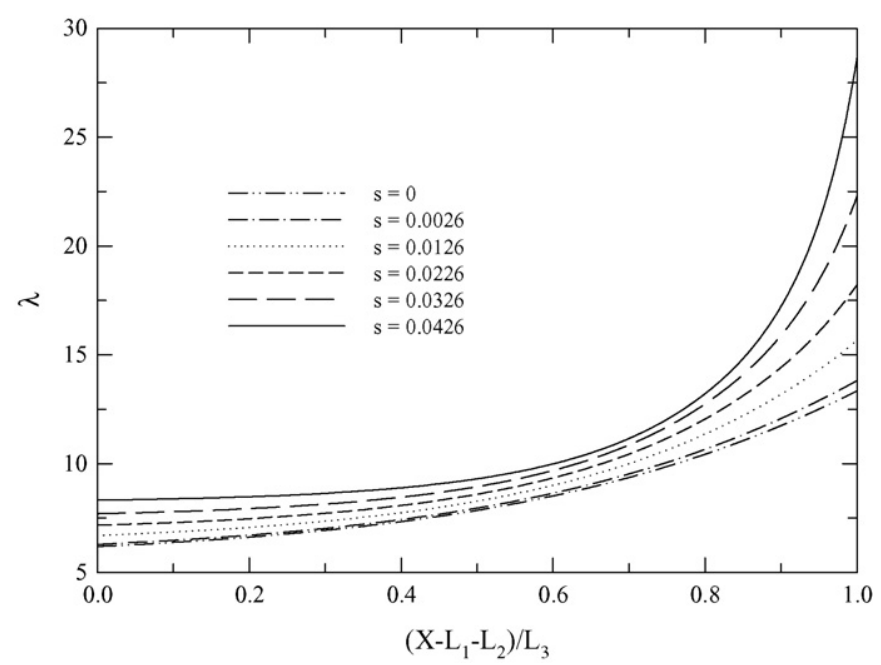

Fig. 6. The profiles of water content in the membrane for several assigned values of membrane expansion coefficient $s$, where $C_{\mathrm{in}, \mathrm{a}} / C_{\mathrm{sat}}=C_{\mathrm{in}, \mathrm{c}} / C_{\mathrm{sat}}=100 \%$, $\varepsilon_{\mathrm{d}}=0.4, L_{3}=175 \mu \mathrm{m}$, and $I=1.0 \mathrm{~A} \mathrm{~cm}^{-2}$.

the water content of membrane $\lambda$ depends on the parameter $s$ especially in the region adjacent to the right cathode interface. The deviation of $\lambda$ at this interface could be approximately $30 \%$ between the case neglecting the effect of membrane swelling with $s=0$ and that with $s=0.0126$, and even higher for larger value of $s$. Hence, the results illustrate that the effect of membrane swelling plays a crucial role in the appearance of flooding phenomenon in the cathode side, and the membrane expansion coefficient $s$ should be as small as possible to preserve its liquid water absorption ability.

The variations of membrane overvoltage $\phi$ with cell current density $I$ are illustrated in Fig. 7 for several typical cases varying from the base state as considered in Fig. 2. In general, the absolute magnitudes of $\phi$ increases gradually with $I$ in all the

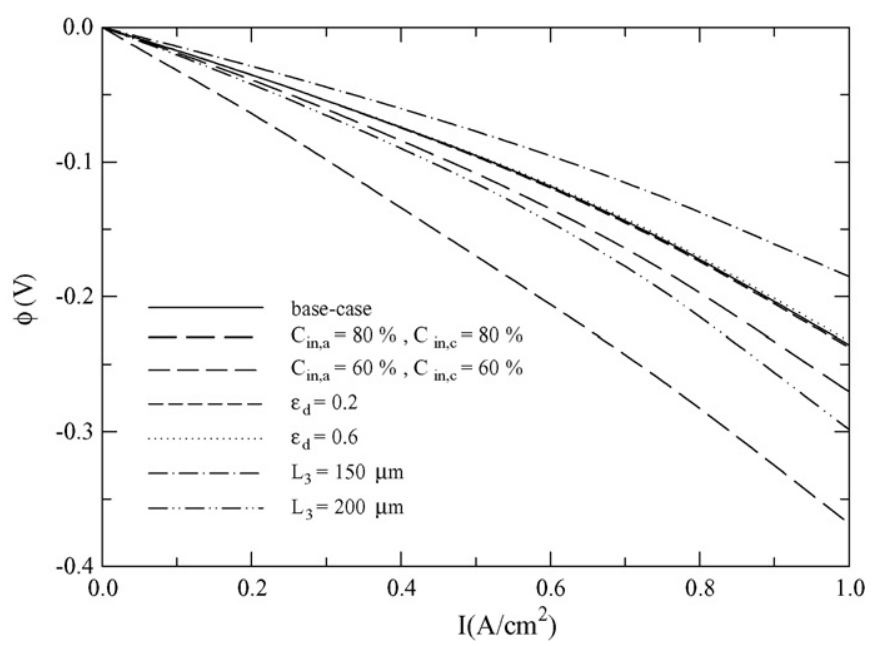

Fig. 7. The variations of membrane overvoltage with the current density for several typical cases deviating from the base case of Fig. 2.

curves presented here. It is found that the membrane overvoltage depends heavily on the relative humidity of reactant gases and the membrane thickness, but is less sensitive to the variation of GDL porosity. A lower water concentration in the intake gases results in a larger membrane overvoltage and this effect grows rapidly with the decrease of humidification since the drying out of the membrane becomes significant. A thicker membrane also increases the loss of membrane overvoltage, and for a fixed value of current density, the overvoltage seems to be proportional to the membrane thickness, which is in agreement with the known requirement that the membrane should be as thin as possible.

The transient performance of a PEMFC is also very important especially in the applications of power sources which have to operate under time-varying load conditions. Fig. 8(a)-(c) shows the variation of water concentration profile in each layer with
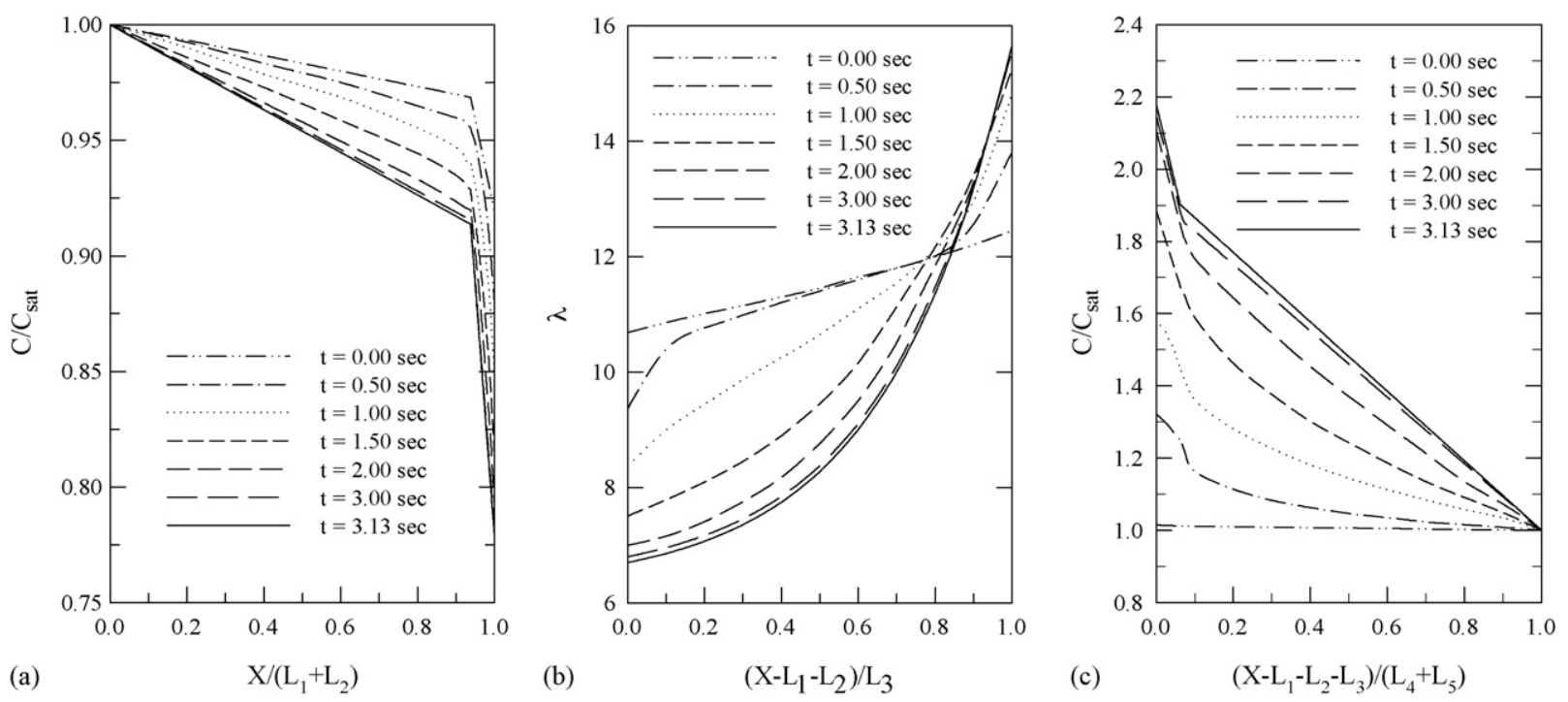

Fig. 8. (a) The transient profiles of water concentration in anode GDL and CL; (b) the transient profiles of water content in the membrane; (c) the transient profiles of water concentration in cathode GDL and CL; with several assigned times when the cell changes from the initial state $I=0.1 \mathrm{~A} \mathrm{~cm}^{-2}$ to the final state $I=1.0 \mathrm{~A} \mathrm{~cm}{ }^{-2}$, where $C_{\mathrm{in}, \mathrm{a}} / C_{\mathrm{sat}}=C_{\mathrm{in,c}} / C_{\mathrm{sat}}=100 \%, \varepsilon_{\mathrm{d}}=0.4, L_{3}=175 \mu \mathrm{m}$, and $s=0.0126$. 


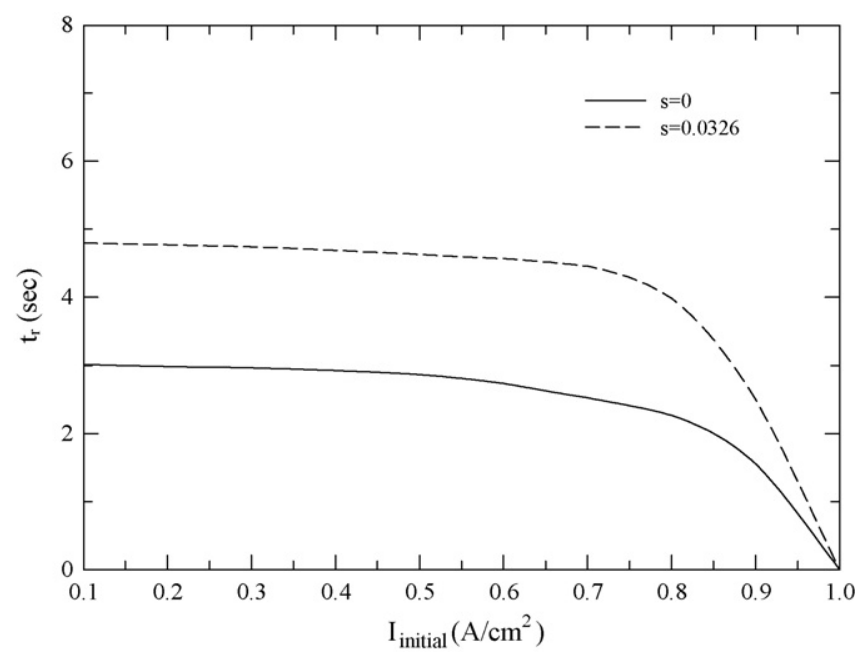

Fig. 9. The response time $t_{\mathrm{r}}$ for a given initial current density $I_{\text {initial }}$ to reach the final steady state $I=1.0 \mathrm{~A} \mathrm{~cm}^{-2}$, where $C_{\mathrm{in,a}} / C_{\mathrm{sat}}=C_{\mathrm{in}, \mathrm{c}} / C_{\mathrm{sat}}=100 \%, \varepsilon_{\mathrm{d}}=0.4$, and $L_{3}=175 \mu \mathrm{m}$.

time from the state $I=0.1 \mathrm{~A} \mathrm{~cm}^{-2}$ to $I=1.0 \mathrm{~A} \mathrm{~cm}^{-2}$. At $t=0$ the initial state with $I=0.1 \mathrm{~A} \mathrm{~cm}^{-2}$, the low reaction rate makes the vapor in the anode gas channel have sufficient time to pass through the anode GDL and CL. In the membrane, the effect of electroosmotic drag is small and the vapor generation rate adjacent to the right interface is low, too. Therefore, the membrane appears to have more uniform water content. The same reason causes the water concentration profiles in the cathode $\mathrm{CL}$ and GDL look flat. As soon as the current density changes to $1.0 \mathrm{~A} \mathrm{~cm}^{-2}$, the higher reaction rate requires higher transport flux. Accordingly, the profile in Fig. 8(a) reduces and that in Fig. 8(c) rises rapidly, and then both approach steady states after time $t=3.13 \mathrm{~s}$. The enhancement of electroosmotic drag makes the water content at the left interface of the membrane lower and that at the right interface rise as shown in Fig. 8(b). The profile also changes quickly at first and then reaches an equilibrium state gradually. Similar results also can be obtained if the relative humidity of intake gases decrease. However, the time for the cell to be steady will be lengthened. For example, it will be 4.12 and $6.23 \mathrm{~s}$, respectively, for the cases of reactant gases with $C / C_{\mathrm{sat}}=80 \%$ and $60 \%$.

Fig. 9 illustrates the membrane swelling effect on the cell transient behaviors. The curves in this figure indicate the response time $t_{\mathrm{r}}$ required for a given initial current density $I_{\text {initial }}$ which is less than $1.0 \mathrm{~A} \mathrm{~cm}^{-2}$ to reach the final state $I=1.0 \mathrm{~A} \mathrm{~cm}^{-2}$. Obviously, the effect of membrane swelling will lengthen the response time. Moreover, the response time seems to be a constant till the initial current density is close to the assigned final state that diminishes gradually to zero.

The influences of relative humidity of intake gases, porosity of GDL, and membrane thickness on the response time are demonstrated in Fig. 10. It is found that the response time is quite sensitive to the variation of membrane thickness and its value becomes larger for a thicker membrane. On the other hand, an increase of GDL porosity may reduce the response time while it plays a less important role in the parameters affecting the transient behavior of the system.

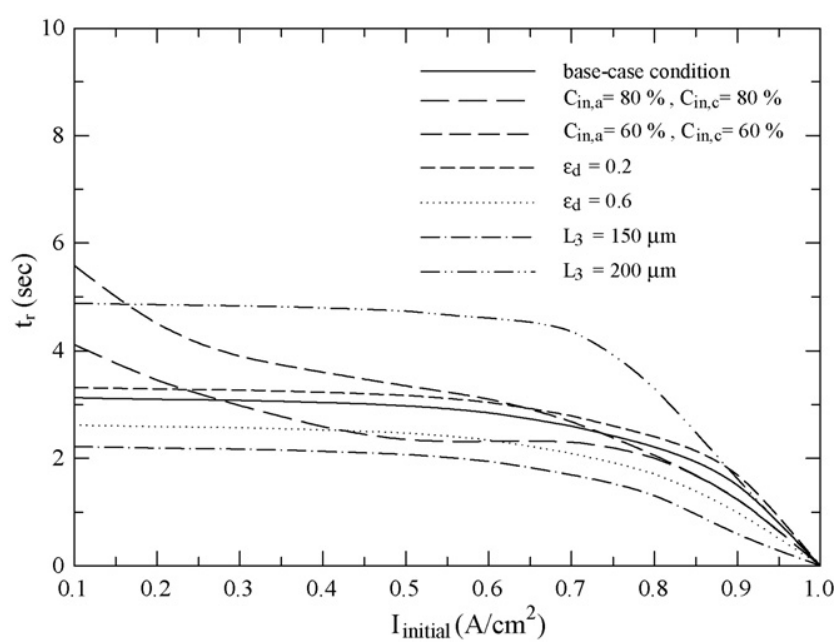

Fig. 10. The variations of response time $t_{\mathrm{r}}$ for a given initial current density $I_{\text {inital }}$ to reach the final steady state $I=1.0 \mathrm{~A} \mathrm{~cm}^{-2}$ for several typical cases deviating from the base case of Fig. 2.

\section{Conclusions}

We have developed a one-dimensional model of water transport for a five-layer PEMFC system. A detailed numerical analysis has been performed to explore the transport characteristics including both steady and transient states. The influences of several crucial system parameters are examined especially the membrane swelling effect. The results show that at the anode side the reactant gas must be humidified with saturated vapor to insure the transport is toward to the membrane in order to avoid the occurrence of drying out of membrane. On the other hand, at the cathode side the saturated intake air causes a supersaturated water concentration in the $\mathrm{CL}$ and thus the cathode takes a risk of flooding problem. It is found that a lower relative humidity about $80 \%$ is better to prevent the flooding phenomenon and keep the membrane with appropriate humidity simultaneously. In the membrane, the water content is dominated by the electroosmotic drag effect at high current density conditions and the value of $\lambda$ at the anodic interface decreases rapidly with current density $I$. Therefore, it is vital to supply more water to the anode side of the membrane at high current density conditions. The results interpret that a higher porosity of GDL or a thinner membrane would be beneficial to enhance the water transport to the anode side of the membrane and maintain the water content there. The membrane expansion coefficient $s$ also plays an important role in the water content profile of the membrane especially at the cathode side. It is found that the water content profile will rise for a membrane with larger value of $s$ and this effect is more pronounced at the cathode interface.

As for the transient analysis, the results show that the response time $t_{\mathrm{r}}$ from one equilibrium state to the other one is related to the relative humidity in the gas channels, the difference of current density, the membrane expansion coefficient, the porosity of $\mathrm{GDL}$, and the membrane thickness. In general, the value of $t_{\mathrm{r}}$ will increase for a larger difference of current density, a higher membrane expansion coefficient, and a thicker membrane; and reduce for higher relative humidity of reactant gases and larger 
porosity of GDL. To improve the transient performance, that is, to shorten the value of $t_{\mathrm{r}}$, the results indicate that the best way is to use a thinner membrane with smaller membrane expansion coefficient. Hence, to make the membrane as thin as possible improves not only the mean water content of the membrane and the induced ohmic losses, but also the transient performance of the system. The present results would be helpful for the design of PEMFC to achieve the optimal control of water transport.

\section{Acknowledgment}

The financial support for this research from National Science Council of Taiwan through the grants NSC 94-2212-E-132005, NSC 94-2212-E-002-008, and NSC 93-2212-E-002-029 are gratefully acknowledged.

\section{References}

[1] T.E. Springer, T.A. Zawodzinski, S. Gottesfeld, J. Electrochem. Soc. 138 (1991) 2334.

[2] D.M. Bernardi, M.W. Verbrugge, AIChE J. 37 (1991) 1151.

[3] D.M. Bernardi, M.W. Verbrugge, J. Electrochem. Soc. 139 (1992) 2477.

[4] T.F. Fuller, J. Newman, J. Electrochem. Soc. 140 (1993) 1218.

[5] T.V. Nguyen, R.E. White, J. Electrochem. Soc. 140 (1993) 2178.

[6] T. Okada, G. Xie, Y. Tanabe, J. Electroanal. Chem. 413 (1996) 49.

[7] V. Gurau, H. Liu, S. Kakac, AIChE J. 44 (1998) 2410.

[8] V. Gurau, F. Barbir, H. Liu, J. Electrochem. Soc. 147 (2000) 2468

[9] I.M. Hsing, P. Futerko, Chem. Eng. Sci. 55 (2000) 4209.

[10] J.J. Baschuk, X. Li, J. Power Sources 86 (2000) 181.

[11] S. Um, C.Y. Wang, K.S. Chen, J. Electrochem. Soc. 147 (2000) 4485.
[12] N. Djilali, D. Lu, Int. J. Therm. Sci. 41 (2002) 29.

[13] A.A. Kulikovsky, J. Electrochem. Soc. 150 (2003) A1432.

[14] N.P. Siegel, M.W. Ellis, D.J. Nelson, M.R. von Spakovsky, J. Power Sources 132 (2003) 127.

[15] C.Y. Wang, Chem. Rev. 104 (2004) 4727.

[16] Y. Wang, C.Y. Wang, Electrochim. Acta 50 (2005) 1307.

[17] Y. Wang, C.Y. Wang, Electrochim. Acta 51 (2006) 3924.

[18] T.A. Zawodzinski Jr., M. Neeman, L.O. Sillerud, S. Gottesfeld, J. Phys. Chem. 95 (1991) 6040.

[19] T.F. Fuller, J. Newman, J. Electrochem. Soc. 139 (1992) 1332.

[20] T.A. Zawodzinski Jr., C. Derouin, S. Radzinski, R.J. Sherman, V.T. Smith, T.E. Springer, S. Gottesfeld, J. Electrochem. Soc. 140 (1993) 1041.

[21] T.A. Zawodzinski Jr., T.E. Springer, J. Davey, R. Jestel, C. Lopez, J. Valerio, S. Gottesfeld, J. Electrochem. Soc. 140 (1993) 1981.

[22] J.T. Hinatsu, M. Mizuhata, H. Takenaka, J. Electrochem. Soc. 141 (1994) 1493.

[23] T.A. Zawodzinski, J. Davey, J. Valerio, S. Gottesfeld, Electrochim. Acta 40 (1995) 297

[24] G. Xie, T. Okada, J. Electrochem. Soc. 142 (1995) 3057.

[25] J. Divisek, M. Eikerling, V. Mazin, H. Schmitz, U. Stimming, Y.M. Volfkovich, J. Electrochem. Soc. 145 (1998) 2677.

[26] M. Eikerling, Y.I. Kharkats, A.A. Kornyshev, Y.M. Volfkovich, J. Electrochem. Soc. 145 (1998) 2684.

[27] T. Okada, G. Xie, M. Meeg, Electrochim. Acta 43 (1998) 2141.

[28] F. Chen, Y.G. Su, C.Y. Soong, W.M. Yan, H.S. Chu, J. Electroanal. Chem. $566(2003) 85$

[29] M.D. Francesco, E. Arato, P. Costa, J. Power Sources 132 (2004) 127.

[30] J. Larminie, A. Dicks, Fuel Cell Systems Explained, John Wiley \& Sons, Chichester, UK, 2000.

[31] R.B. Bird, W.E. Stewart, E.N. Lightfoot, Transport Phenomena, Wiley, New York, 1960.

[32] G. Lin, W. He, T.V. Nguyen, J. Electrochem. Soc. 151 (2004) A1999. 